\title{
Fatores de risco para COVID-19 em idosas atendidas em consultórío de enfermagem
}

\author{
Risk factors for COVID-19 in elderly care in a nursing consulting \\ Factores de riesgo de COVID-19 en cuidados de ancianos en consultoría de enfermería
}

\begin{abstract}
RESUMO
Objetivo: Descrever os fatores de risco para COVID-19 de idosas atendidas em um Consultório de Enfermagem. Método: Pesquisa descritiva, quantitativa, utilizando dos dados contidos nos prontuários das mulheres atendidas no Consultório de Enfermagem de uma Universidade Pública do estado do Rio de Janeiro, de 2017 a 2019. Resultados: Analisados 303 prontuários, revelando que $25,2 \%$ possuem 60 anos ou mais. Destas, $51,2 \%$ são solteiras e $45,7 \%$ brancas. Em relação às doenças crônico-degenerativas $29,7 \%$ das mulheres idosas possuem doenças crônicas como Hipertensão Arterial (61,5\%), Diabetes Mellitus (9,6\%) e Câncer (9,6\%). 0 sobrepeso incidiu em $42,1 \%$ e, 62,5\% aderiram à imunização para influenza no último ano. Conclusão: Constatou-se que as mulheres idosas atendidas no consultório possuem fatores de risco para COVID-19, tais como: sobrepeso, doenças crônico-degenerativas e baixa adesão para imunização. Devemos fortalecer a conscientização sobre a gravidade da doença com ações de promoção da saúde e prevenção da doença COVID-19.
\end{abstract}

DESCRITORES: Comorbidades; Saúde da Mulher; COVID-19; Promoção da Saúde; Consulta de Enfermagem.

\section{ABSTRACT}

Objective: To describe the risk factors for COVID-19 of elderly women seen in a Nursing Consulting. Method: Descriptive, quantitative research, using the data contained in the medical records of users seen at the Nursing Office of a Public University of the State of Rio de Janeiro, from 2017 to 2019. Results: 303 medical records were analyzed, revealing that $25.2 \%$ are 60 years old or older. Of these, $51.2 \%$ are single and $45.7 \%$ white. Regarding chronic-degenerative diseases, $29.7 \%$ of elderly women have chronic diseases, Arterial Hypertension (61.5\%), Diabetes Mellitus (9.6\%) and Cancer (9.6\%). Overweight occurred in $42.1 \%$ and $62.5 \%$ adhered to influenza immunization in the last year. Conclusion: It was found that elderly women seen in the office have risk factors for COVID-19, such as: overweight, chronic degenerative diseases and low adherence to immunization. We must strengthen awareness about the severity of the disease with health promotion and disease prevention actions COVID-19.

DESCRIPTORS: Comorbidities; Women's Health; COVID-19; Health Promotion; Office Nursing.

\section{RESUMEN}

Objetivo: Describir los factores de riesgo de COVID-19 en mujeres ancianas atendidas en la Oficina de Enfermería. Método: Investigación descriptiva, cuantitativa, utilizando los datos contenidos en las historias clínicas de los usuarios atendidos en la Oficina de Enfermería de una Universidad Pública del estado de Rio de Janeiro, de 2017 a 2019. Resultados: se analizaron 303 historias clínicas, revelando que el $25,2 \%$ tiene 60 años o más. De estos, el $51,2 \%$ son solteros y el $45,7 \%$ blancos. En cuanto a las enfermedades crónico-degenerativas, el 29,7\% de las mujeres mayores tienen enfermedades crónicas, Hipertensión Arterial (61,5\%), Diabetes Mellitus (9,6\%) y Cáncer (9,6\%). El 42,1\% presentó sobrepeso y el 62,5\% se adhirió a la vacunación antigripal en el último año. Conclusión: Se encontró que las ancianas atendidas en consultorio presentan factores de riesgo para COVID-19, como: sobrepeso, enfermedades crónico degenerativas y baja adherencia a la inmunización. Debemos fortalecer la conciencia sobre la gravedad de la enfermedad con acciones de promoción de la salud y prevención de enfermedades COVID-19.

DESCRIPTORES: Comorbilidades; La Salud de la Mujer; COVID-19; Promoción de la Salud; Enfermería de Consulta.

RECEBIDO EM: 29/03/2021 APROVADO EM: 15/04/2021

\section{Brenda Freitas Pontes}

Acadêmica de Enfermagem. Universidade Federal Fluminense/Campus Rio das Ostras. Bolsista Iniciação Científica/PIBIC/ UFF/CNPq.

ORCID: 0000-0001-9246-4395 


\section{Rosana de Carvalho Castro}

Enfermeira Obstétrica. Doutora em Enfermagem pela EEAN/UFRJ. Professora Adjunta do Departamento de Enfermagem Universidade Federal Fluminense/Campus Rio das Ostras. Vice-líder do Grupo de Pesquisa: Laboratório de Estudos sobre Mulheres e Enfermagem (LEME).

ORCID: 0000-0003-0379-9244

\section{Jane Baptista Quitete}

Enfermeira Obstétrica. Doutora em Enfermagem pela UERJ. Professora Adjunta do Departamento de Enfermagem Universidade Federal Fluminense/Campus Rio das Ostras. Líder do Grupo de Pesquisa: Laboratório de Estudos sobre Mulheres e Enfermagem (LEME).

ORCID: 0000-0003-0330-458X

\section{Mariana Tavares da Silva}

Acadêmica de Enfermagem. Universidade Federal Fluminense/Campus Rio das Ostras.Bolsista Iniciação Científica/PIBIC. ORCID: 0000-0002-8862-2474

\section{Sthefany Suzana Dantas da Silveira}

Acadêmica de Enfermagem. Universidade Federal Fluminense/Campus Rio das Ostras.Bolsista do Programa de Educação Tutorial/ PET Conexões de Saberes Enfermagem.

ORCID: 0000-0002-1171-600X

\section{Beatriz Garcia de Souza}

Acadêmica de Enfermagem. Universidade federal Fluminense/Campus Rio das Ostras.

ORCID: 0000-0002-4071-4601

\section{INTRODUÇÃO}

$\mathbf{N}$ o Brasil, cerca de 6,76\% da população brasileira são de mulheres idosas segundo o $\mathrm{IBGE}^{1}$ e, cerca de $60 \%$ dos idosos no Brasil, possuem hipertensão arterial sistêmica (HAS) e diabetes mellitus $(\mathrm{DM})^{2}$ Estudos demostram que idosos e pessoas portadoras de doenças crônico-degenerativas, obesidade ou câncer, são mais propícias ao risco de óbito por COVID-193.

O COVID-19 é causado por um novo coronavírus denominado SARS-CoV-2. O coronavírus pertence à família Coronaviridae que causam infecções respiratórias, é um vírus zoonótico, possui um RNA vírus da ordem Nidovirales ${ }^{4,5}$. São diversas espécies de vírus comuns em diferentes tipos de animais, sendo eles morcegos, gado, gatos e camelos. Em dezembro de 2019 houve o contagio e transmissão de um novo coronavírus (SARS-CoV-2), identificado em Wuhan na China ocasionando a COVID-19 e disseminando a doença no mundo inteiro ${ }^{6}$. Os tipos de coronavírus conhecidos atualmente são: alfa coronavírus $\mathrm{HCoV}-229 \mathrm{E}$ e alfa coronavírus $\mathrm{HCo}-$ V-NL63, beta coronavírus HCoV-OC43 e beta coronavírus HCoV-HKU1, SARS- $\mathrm{CoV}$ (causador da síndrome respiratória aguda grave ou SARS), MERS-CoV (causador da síndrome respiratória do Oriente Médio ou MERS) e SARS-CoV-2 (causador do COVID-19)7. A transmissão do SARS-CoV-2 ocorre por contato de gotículas respiratórias de pacientes contaminados o COVID-19 afeta principalmente os sistemas respiratório, cardiovascular, gastrointestinal e neurológico ${ }^{6}$.

Segundo a Organização Mundial da Saúde o número de casos de COVID-19 no mundo até 12 de Abril de 2021 foi de 135646617 casos confirmados e 2930 732 mortes $^{8}$. A Organização Pan-Americana da Saúde (OPAS) documentou o aumento de $14 \%$ nos casos e de $14 \%$ nas mortes nas Américas de 15 de janeiro a 8 de fevereiro de $2021^{8}$. Três variantes do vírus SARS-CoV-2 em circulação nas Américas que mais preocupam: $\mathrm{SARS}-\mathrm{CoV}-2$ VOC 202012/01 (Reino Unido), 501Y. V2 (África do Sul) e B.1.1.2810 (Brasil) ${ }^{9}$ . Estados Unidos três variantes, enquanto Argentina, Brasil, Canadá e Peru registraram duas delas. A OPAS afirma que Américas devem ser "prioridade global" para as vacinas contra a COVID-19, .
Tendo em vista as especificidades desta nova infecção e a necessária intervenção em promoção e proteção da saúde, o objetivo deste trabalho é descrever os fatores de risco para COVID-19 de idosas atendidas no Consultório de Enfermagem de uma Universidade Pública.

\section{MÉTODO}

Trata-se de uma pesquisa descritiva, de natureza quantitativa, tendo como cenário o Consultório de Enfermagem de uma Universidade Pública do estado do Rio de Janeiro. A população do estudo foi composta por mulheres idosas atendidas no Consultório de Enfermagem no período de 2017 a 2019.

Coleta de dados realizada de agosto de 2019 a novembro de 2020 acessando os prontuários das mulheres atendidas. Os critérios de inclusão foram: os prontuários de usuárias idosas do sexo feminino. Os critérios de exclusão serão: prontuários do sexo masculino e prontuários incompletos. Os dados coletados foram digitados em planilha eletrônica e processados no Programa R, que é gratuito e disponível para download. ${ }^{10}$ Foi calculada a proporção e as medidas de tendência central. 


\section{Quadro 01: Caracterização sócio demográfica} VARIÁVEIS

\section{Idade}

- 10 a 19 anos (adolescente)

- 20 a 39 anos (adulto jovem)

- 40 a 59 anos (meia idade)

-60 anos ou mais

- 60 anos - 69 anos

- 70 anos - 79 anos

- 80 anos - 89 anos

- Acima de 90 anos

Estado civil

- Solteira

- Casada

- União estável

- Divorciada

- Viúvas

Cor autodeclarada

- Amarela

- Branca

- Parda

- Preta

- Não declarada

Escolaridade

- Analfabeto

- Fundamental Incompleto

- Fundamental completo

- Médio Incompleto

- Médio completo

- Superior Incompleto

- Superior completo

- Doutorado

Fonte: As autoras.

Quadro 02: Histórico de saúde e histórico familiar com relação às doenças crônico-degenerativa

\section{HISTÓRICO DE SAÚDE COM RELAÇÃO ÀS DOENÇAS CRÔNICO-} DEGENERATIVA

\section{- Possuem}

- Não possuem

Histórico de saúde com relação às doenças crônico-degenerativa

- Hipertensão Arterial Sistêmica

- Diabettes

\begin{tabular}{l|l}
\hline $\mathbf{N}$ & \%
\end{tabular}

9,0

30,2

42

25,2

17

4,6

0

0

152

51,2

$86 \quad 29$

25

8,4

93

25

8,4

$25-8,4$

5

3

\begin{tabular}{l|l}
74 & 45,7
\end{tabular}

45

27,8

$35 \quad 21,6$

3

1,9

$3 \quad 1,9$

2

0,7

39

13,4

$24 \quad 8,2$

14

4,2

98

33,7

\begin{tabular}{l|l}
79 & 27,1 \\
\hline
\end{tabular}

34

11,7

0,3

1

(1)

(2)




\begin{tabular}{|l|c|c|}
\hline - Câncer & 5 & 9,6 \\
\hline - Outros & 20 & 49,4 \\
\hline Histórico familiar com relação às doenças crônico-degenerativa & $\mathrm{N}$ & $\%$ \\
\hline - Hipertensão Arterial Sistêmica & 193 & 73,9 \\
\hline - Diabettes & 138 & 52,9 \\
\hline - Câncer & 157 & 60,2 \\
\hline - Outros & 12 & 4,8 \\
\hline Fonte: As autoras. & \multicolumn{3}{|l|}{} \\
\hline
\end{tabular}

Quadro 2: Avaliação em saúde

\begin{tabular}{|l|c|c|}
\hline IMC & N & $\%$ \\
\hline - Peso normal: IMC entre 18.0 a 24,9 & 29 & 22,4 \\
\hline - Sobrepeso: IMC entre 25.0 a 29,9 & 22 & 17,5 \\
\hline - Obesidade grau 1: IMC entre 30.0 a 34.9 & 14 & 11,1 \\
\hline - Obesidade grau 2: IMC entre 35.0 a 39.9 & 12 & 9,5 \\
\hline - Obesidade grau 3 ou obesidade mórbida: IMC igual ou superior 40 & 5 & 4 \\
\hline Fonte: As autoras. &
\end{tabular}

\section{Quadro 3: Estado vacinal}

\begin{tabular}{|l|c|c|}
\hline ESTADO VACINAL & $\mathbf{N}$ & $\%$ \\
\hline - Vacinaram-se para a gripe & 163 & 62,5 \\
\hline - Não vacinaram-se & 98 & 37,5 \\
\hline Fonte: As autoras. & \multicolumn{2}{l}{} \\
\hline
\end{tabular}

da população idosa e elevação da expectativa de vida. Ao longo das últimas décadas ocorreram mudanças demográficas e epidemiológicas como diminuição das doenças infecciosas, aumento das doenças crônicas, transição nutricional e aumento na expectativa de vida. ${ }^{11}$

Com relação ao histórico pessoal de saúde referente às doenças crônico-degenerativa (HAS, DM, Câncer) associada ao envelhecimento, o COVID-19 manifesta-se mais agressivamente elevando o risco da presença de manifestações sistêmicas, como a Síndrome Respiratória Aguda Grave (SRAG) e falência de múltiplos órgãos. ${ }^{12}$ Estudos revelam que idade avançada e fragilidade são os principais preditores de mortalidade em pacientes com COVID-19 internados ${ }^{12}$.

Ademais, a presença de doenças crônico-degenerativas tem ligação com a patogênese da COVID-19. ${ }^{11}$ Estudos internacionais também tem demonstrado que a faixa etária tem interferência e ligação para

\section{Ao longo das}

últimas décadas

ocorreram mudanças

demográficas e

epidemiológicas

como diminuição das

doenças infecciosas,

aumento das doenças

crônicas, transição

nutricional e aumento

na expectativa de vida. desfechos desfavoráveis como internação hospitalar, necessidade de Unidade de Tratamento Intensivo (UTI) e óbito. ${ }^{13,14}$ Este patógeno muitas vezes leva a uma síndrome que pode levar a condição respiratória de terapia intensiva, sendo necessário tratamento especializado em unidades de terapia intensiva (UTI) $1^{3,14,15}$ A taxa de letalidade entre pacientes hospitalizados é superior a $10 \%^{13}$. Obesidade e as neoplasias também estão associadas a elevação das taxas de letalidade ${ }^{16,17,18}$.

O estudo de (Jing Yang et al, 2020) na China com dados de aproximadamente 45.000 pacientes evidenciou que hipertensão(HAS) (17\%), diabetes(DM) (8\%), doenças cardiovasculares (5\%) e doenças respiratórias crônicas (2\%) eram as doenças apresentadas com mais frequência em pacientes com risco elevado de óbito. ${ }^{15} \mathrm{~A}$ HAS é descrita por níveis elevados e sustentados de pressão arterial ( $\mathrm{PA} \geq 140 \mathrm{x}$ $90 \mathrm{mmHg}$ ). É uma condição clinica causada por diversos fatores, e está associada a distúrbios funcionais e estruturais dos órgãos como coração, encéfalo, rins e vasos sanguíneos e às alterações metabólicas. Esta patologia possui alta taxa morbimortalidade, o que reforça a importância do diagnóstico precoce $^{11}$. A HAS condiciona associação independente com ocorrências de morte súbita, acidente vascular encefálico (AVE), infarto agudo do miocárdio (IAM), insuficiência cardíaca (IC), doença arterial periférica (DAP) e doença renal crônica (DRC), fatal e não fatal. ${ }^{19}$ Diabetes Mellitus (DM) é um transtorno metabólico de etiologias heterogêneas, caracterizado por hiperglicemia e distúrbios no metabolismo de carboidratos, proteínas e gorduras, resultantes de defeitos da secreção e/ou da ação da insulina ${ }^{20}$. Fatores como rápida urbanização, transição epidemiológica, transição nutricional, maior frequência de estilo de vida sedentário, maior frequência de excesso de peso, crescimento e envelhecimento populacional e, também, à maior sobrevida dos indivíduos com diabetes elevam a prevalência da DM. ${ }^{21}$

Referente ao Índice de Massa Corporal (IMC), estudos internacionais relatam a intensa relação da obesidade em pacientes 
graves internados em terapia intensiva por SARS-CoV-2, devendo-se, assim, levar em conta o fato das comorbidades associadas ao COVID-19, como o DM, muitas vezes serem consequências da obesidade. ${ }^{22,23}$ A obesidade é um dos fatores de riscos as doenças e agravos não transmissíveis, logo, a prevenção e diagnóstico precoce são extremamente relevantes para promoção da saúde e redução de morbimortalidade ${ }^{24}$. Nota-se poucos estudos referentes a dimensão problemática da saúde mental. ${ }^{25}$

No Brasil, as vacinas Oxford e Coronavac, foram aprovadas pela Agência Nacional de Vigilância Sanitária (ANVISA) em janeiro de 2021 para uso emergencial ${ }^{26}$. A vacina da Janssen foi adicionada à lista de ferramentas de emergência seguras e eficazes contra COVID-19 pela OMS ${ }^{27}$. Ou seja, o uso emergencial significa que não foram finalizadas todas as etapas da pesquisa, mas há resultados suficientes que garantem eficácia e segurança à população. O COVAX (Acesso Global às Vacinas da Covid-19) um esforço global da Coalizão para Promoção de Inovações em prol da Preparação para Epidemias (CEPI), da Aliança Mundial para Vacinas e Imunização (Gavi), do Fundo das Nações Unidas para a Infância (UNICEF), da Organização Mundial da Saúde (OMS) e da Organização Pan-Americana da Saúde (OPAS). O Brasil receberá doses da vacina AstraZeneca/Oxford - fabricada pelo SK Bioscience, da Coreia do Sul.contra COVID-19 através desse meio ${ }^{28}$. A imunização protege o indivíduo e os que estão ao seu redor e salva milhões de vidas todos os anos, reduzindo o risco de contrair doença e proporcionando proteção ${ }^{26,27}$.

\section{Constatou-se}

que as mulheres

idosas atendidas

no Consultório

de Enfermagem

possuem fatores

de risco para

COVID-19, tais

como: sobrepeso,

doenças crônico

degenerativas e

baixa adesão para

imunização.

Apesar de pesquisas e comprovações inegáveis que revelam a eficácia e importância da vacina, cresce o número de pessoas que se recusam a se vacinar e vacinar seus filhos, formando o movimento anti-vacina, fato preocupante, pois as vacinas são produzidas em um rigoroso processo de produção, contanto com estudos e pesquisas e passam por inúmeros testes até serem validadas. ${ }^{29} \mathrm{O}$ Brasil vem passando por uma redução preocupante na cobertu- ra vacinal nos últimos anos ${ }^{29}$ Especialistas consideram, como um dos principais motivos da baixa cobertura vacinal, o modelo de atenção à saúde atual no País, que prioriza as condições agudas de saúde e que não dá conta do devido acompanhamento dos cidadãos. Ações de vigilância, prevenção e promoção são fundamentais e nem sempre a organização dos serviços de saúde privilegia a continuidade do cuidado. ${ }^{29}$

\section{CONCLUSÃO}

Constatou-se que as mulheres idosas atendidas no Consultório de Enfermagem possuem fatores de risco para $\mathrm{CO}$ VID-19, tais como: sobrepeso, doenças crônico degenerativas e baixa adesão para imunização. Conhecer o perfil clínico, doenças crônicas como Hipertensão Arterial (61,5\%) e Diabetes Mellitus (9,6\%), e epidemiológico, são solteiras $(51,2 \%)$ e brancas $(45,7 \%)$, desta população permitiu inferir as necessidades de saúde, possibilitando o planejamento e a implementação de cuidados de enfermagem.

Deste modo, devemos fortalecer a conscientização da população de mulheres idosas sobre a gravidade da doença com ações de promoção da saúde com vistas ao autocuidado, prevenção de doenças, alimentação saudável e atividade física. Bem como, estimular a adesão à imunização da população contra a COVID e medidas de saúde pública de prevenção, proteção e controle como distanciamento social, lavagem das mãos com frequência e cobrir a boca com um lenço de papel ou cotovelo dobrado ao espirrar ou tossir e uso de uma máscara de tecido preconizadas pela $\mathrm{OMS}^{3}$.

\section{REFERÊNCIAS}

1. Instituto Brasileiro de Geografia e estatística. (IBGE, 2008). [Acesso em 12 Abr 2021] Disponivel em: https://www.ibge.gov. br/apps/populacao/projecao/

2. Ministério da Saúde. (BRASIL). Secretaria de atenção primária a saúde. 2020. [Acesso em 12 Abr 2021] Disponivel em: https:// aps.saude.gov.br/noticia/10018\#: :text=Atualmente $\% 2 C \% 20$ no\%20Brasil\%2C\%20a\%20popula\%C3\%A7\%C3\%A3o,(Vigitel) $\% 2$ C $\% 20$ de $\% 202018$.
3. Organização Pan-Americana de Saúde (OPAS/OMS, 2020). [Acesso em 20 Jan 2021]. Disponivel em: https://www.who.int/ news-room/q-a-detail/coronavirus-disease-covid-19

4. Ministério da Saúde (Brasil). Protocolo de manejo clínico para o novo-coronavírus (2019-nCoV). [recurso eletrônico] Acesso em 2021 Jan 20. Disponivel em: https://portalarquivos2.saude.gov. br/images/pdf/2020/fevereiro/11/protocolo-manejo-coronavirus.pdf. 


\section{REFERÊNCIAS}

5. Ministério da Saúde (Brasil). Coronavírus: o que você precisa saber e como prevenir o contágio. 2020 Acesso em: 2021 Jan 20. Disponível em: https://saude.gov.br/saude-de-a-z/coronavírus

6. Ministério da Saúde (Brasil). Sobre a doença. O que é o COVID-19. [Internet]. 2020. Acesso em 20 jan 2021. Disponível em: https://coronavirus.saude.gov.br/sobre-a-doenca\#o-que-ecovid

7. Sanders JM, Monogue ML, Jodlowski TZ, Cutrell JB. Pharmacologic Treatments for Coronavirus Disease 2019 (COVID-19): A Review. JAMA [Internet]. 13 de abril de 2020 [citado 6 de maio de 2020]; Disponível em: https://jamanetwork.com/journals/jama/ fullarticle/2764727

8. Organização Pan-Americana de Saúde (OPAS/OMS, 2021). [Acesso em 20 Jan 2021]. Disponível em: https://www.paho.org/ pt/noticias/11-2-2021-casos-covid-19-nas-americas-aumentaram-14-desde-15-janeiro-mas-numero-e-menor

9. Organização Pan-Americana de Saúde (OPAS/OMS, 2020). [Acesso em 20 Jan 2021]. Disponivel em: https://www.paho.org/ bra/index.php?option=com_content\&view=article\&id=6101:covid19\&Item id=875.

10. Programa R. R Foundation for statistical computing. Disponível em: https://cran.r-project.org/mirrors.html.

11. Ministério da Saúde (Brasil). Estratégias para o cuidado da pessoa com doença crônica: hipertensão arterial sistêmica. Brasília: Ministério da Saúde, 2013, Secretaria de Atenção à Saúde, Departamento de atenção básica.

12. Chinnadurai, R., Ogedengbe, O., Agarwal, P. et al. Idade avançada e fragilidade são os principais preditores de mortalidade em pacientes com COVID-19 internados em uma unidade médica de agudos em um ambiente de atenção secundária - um estudo de coorte. BMC Geriatr 20, 409 (2020).

13. Rodriguez-Morales AJ, Cardona-Ospina JA, Gutiérrez-Ocampo $E$, et al. Clinical, laboratory and imaging features of COVID-19: A systematic review and meta-analysis. Travel Med Infect Dis. 2020;34:101623.

14. Nunes, B, Souza ASS, Nogueira J. Andrade FB, Thumé E. et al Envelhecimento, multimorbidade e risco para COVID-19 grave: ELSI-Brasil. Scielo Preprint (Pilot). Disponivel em: https://preprints.scielo.org/index.php/scielo/preprint/view/703/929 Acesso em: 22 mar. 2021.

15. Yang J, Zheng Y, Gou X, Pu K, Chen Z, Guo Q, et al. Prevalence of comorbidities and its effects in patients infected with SARSCoV-2: a systematic review and meta-analysis. Int J Infect Dis [Internet].;94:91-5. Acesso em: 22 mar 2021 Disponivel em: https://www.ijidonline.com/article/S1201-9712(20)30136-3/ abstract

16. Wu Z, McGoogan JM. Characteristics of and Important Lessons From the Coronavirus Disease 2019 (COVID-19) Outbreak in China: Summary of a Report of 72314 Cases From the Chinese Center for Disease Control and Prevention. JAMA [Internet].;323(13):1239-42. Acesso em: 22 mar 2021 Disponível em: https://jamanetwork.com/journals/jama/fullarticle/2762130
17. Halstead SB, Katzelnick L. COVID-19 Vaccines: Should We Fear ADE? J Infect Dis. 2020 Nov 13;222(12):1946-1950

18. Lippi G, Mattiuzzi C, Sanchis Gomar F, Henry BM. Clinical and demographic characteristics of patients dying from COVID 19 in Italy versus China. J Med Virol [Internet]. 10 de abril de 2020

19. Malachias MVB, Souza WKSB, Plavnik FL, Rodrigues CIS, Brandão AA, Neves MFT, et al. 7a Diretriz Brasileira de Hipertensão Arterial. Arq Bras Cardiol 2016; 107(3Supl.3):1-83

20. Ministério da Saúde (Brasil). Estratégias para o cuidado da pessoa com doença crônica: diabetes mellitus. Brasília: Ministério da Saúde, 2013. (Cadernos de Atenção Básica, n. 36)

21. SBD - Sociedade Brasileira de Diabetes. Diretrizes da Sociedade Brasileira de Diabetes: 2019-2020. São Paulo: Clannad; 2019.

22. Bolsoni-Lopes A, Furieri L, Alonso-Vale MIC. Obesidade e a covid-19: uma reflexão sobre a relação entre as pandemias. Rev Gaúcha Enferm. 2021;42(esp):e20200216.

23. Simonnet A, Chetboun M, Poissy J, Raverdy V, Noulette J, Duhamel A, Labreuche J, Mathieu D, Pattou F, Jourdain M; LICORN and the Lille COVID-19 and Obesity study group. High Prevalence of Obesity in Severe Acute Respiratory Syndrome Coronavirus-2 (SARS-CoV-2) Requiring Invasive Mechanical Ventilation. Obesity (Silver Spring). 2020 Jul;28(7):1195-1199. doi: 10.1002/ oby.22831. Epub 2020 Jun 10. Erratum in: Obesity (Silver Spring). 2020 Oct;28(10):1994. PMID: 32271993; PMCID: PMC7262326.

24. Ministério da Saúde (Brasil). Estratégias para o cuidado da pessoa com doença crônica: obesidade / Ministério da Saúde, Secretaria de Atenção à Saúde, Departamento de atenção básica. Brasília - DF, 2017.

25. Araújo MHN, Regis MZM, Araújo SA, Silva RKP, Fernandes DMAP, Melo VFC. Ferramentas utilizadas no cuidado continuado em saúde mental no contexto da COVID-19: uma revisão integrativa. Revista saúde coletiva. 2021; (11) N.62

26 Agência Nacional de Vigilância Sanitária (Brasil). Vacina contra Covid-19: dos testes iniciais ao registro. [Internet]. 2020. Acesso em 20 Jan 2021. Disponivel em: https://www.gov.br/anvisa/ pt-br/assuntos/noticias-anvisa/2020/vacina-contra-covid-19dos-testes-iniciais-ao-registro

27. Organização Pan-Americana de Saúde (OPAS/OMS, 2021). [Acesso em 20 Jan 2021]. Disponivel em: https://www. who.int/news-room/q-a-detail/vaccines-and-immunization-what-is-vaccination?adgroupsurvey=\{adgroupsurvey $\}$ \&gclid=CjwKCAiAxp-ABhALEiwAXm6lyX23iesqTgaQhxYikNEiJl6V7HCCFiTvKSxmFpxi4DHQnRgXJDPRoCJyOQAvD_BwE

28. Organização Pan-Americana de Saúde (OPAS/OMS, 2021). [Acesso em 20 Jan 2021]. Disponível em: https://www.paho. org/pt/noticias/21-3-2021-brasil-recebera-primeiras-vacinascontra-covid-19-por-meio-do-mecanismo-covax

29. Adriane Cruz. A queda da imunização no Brasil. "Como e Por Que as Desigualdades Sociais Fazem Mal à Saude: e-book interativo", Editora Fiocruz. 2017 